\title{
Tensor renormalization group study of two-dimensional $U(1)$ lattice gauge theory with a $\theta$ term
}

\author{
Yoshinobu Kuramashi and Yusuke Yoshimura \\ Center for Computational Sciences, University of Tsukuba, \\ Tsukuba, Ibaraki 305-8577, Japan \\ E-mail: kuramasi@het.ph.tsukuba.ac.jp, yoshimur@ccs.tsukuba.ac.jp
}

AbStRACT: We make an analysis of the two-dimensional $\mathrm{U}(1)$ lattice gauge theory with a $\theta$ term by using the tensor renormalization group. Our numerical result for the free energy shows good consistency with the exact one at finite coupling constant. The topological charge density generates a finite gap at $\theta=\pi$ toward the thermodynamic limit. In addition finite size scaling analysis of the topological susceptibility up to $V=L \times L=1024 \times 1024$ allows us to determine the phase transition at $\theta=\pi$ is the first order.

KeYwords: Lattice QCD, Lattice Quantum Field Theory

ARXIV EPRINT: 1911.06480 


\section{Contents}

1 Introduction 1

2 Tensor renormalization group algorithm $\quad 2$

$2.12 \mathrm{D}$ pure $\mathrm{U}(1)$ lattice gauge theory with a $\theta$ term 2

2.2 Gauss-Legendre quadrature method 3

2.3 Improved method 3

3 Numerical analysis $\quad 4$

3.1 Setup 4

3.2 Free energy 4

3.3 Topological charge density 5

$\begin{array}{lll}3.4 & \text { Topological susceptibility } & 6\end{array}$

4 Summary and outlook $\quad 8$

\section{Introduction}

It has been argued that pure gauge theories with a $\theta$ term contain intriguing nonperturbative aspects. Possible phase transition in the two-dimensional $(2 \mathrm{D})$ pure $\mathrm{U}(N)$ gauge theory was investigated at $\theta=0$ in the large $N$ limit by Gross and Witten thirty years ago [1] and Seiberg discussed that it has a phase transition at $\theta=\pi$ in the strong coupling limit [2]. Later Witten showed that the four-dimensional (4D) pure Yang-Mills theory yields the spontaneous $\mathrm{CP}$ violation at $\theta=\pi$ in the large $N$ limit [3]. Recently this nontrivial phenomena was also predicted based on the argument of the anomaly matching between the CP symmetry and the center symmetry [4]. Up to now, unfortunately, the numerical study with the lattice formulation has not been an efficient tool to investigate these nonperturbative phenomena. The reason is that the lattice numerical methods are based on the Monte Carlo algorithm so that they suffer from the sign problem caused by the introduction of the $\theta$ term.

In 2007 the tensor renormalization group (TRG) was proposed by Levin and Nave to study 2D classical spin models [5]. They pointed out that the TRG method does not suffer from the sign problem in principle. This is a fascinating feature to attract the attention of the elementary particle physicists, who have been struggling with the sign problem to investigate the finite density $\mathrm{QCD}$, the strong $\mathrm{CP}$ problem, the lattice supersymmetry and so on. In past several years exploratory numerical studies were performed by applying the TRG method to the quantum field theories in the path-integral formalism [6-19]. The authors and their collaborators have confirmed that the TRG method is free from the sign problem by successfully demonstrating the phase structure predicted by Coleman [20] for 
the one-flavor Schwinger model with the $\theta$ term employing the Wilson fermion formulation $[8]^{1}$ and the Bose condensation accompanied with the Silver Blaze phenomena in the $2 \mathrm{D}$ complex scalar $\phi^{4}$ theory at the finite density [19].

In this article we apply the TRG method to the $2 \mathrm{D}$ pure $\mathrm{U}(1)$ lattice gauge theory with a $\theta$ term. Since this is the simplest pure lattice gauge theory with a $\theta$ term and the analytical result for the partition function is already known [22], it is a good test case for the TRG method to check the feasibility to investigate the nonperturbative properties of the lattice gauge theories with a $\theta$ term. In the previous studies of Schwinger model with and without the $\theta$ term [7-9], we employed the character expansion method to construct the tensor network representation following the proposal in ref. [23]. In this work, however, we use the Gauss quadrature method with some improvement to discretize the phase in the $\mathrm{U}(1)$ link variable. This is motivated by the success of the Gauss quadrature method to discretize the continuous degree of freedom in the TRG studies of the scalar field theories $[16,19]$.

This paper is organized as follows. In section 2 we explain the TRG method with the use of the Gauss quadrature to calculate the partition function of the 2D pure $\mathrm{U}(1)$ gauge theory. Numerical results for the phase transition at $\theta=\pi$ are presented in section 3 , where our results are compared with the exact ones which are analytically obtained. Section 4 is devoted to summary and outlook.

\section{Tensor renormalization group algorithm}

\subsection{D pure $\mathrm{U}(1)$ lattice gauge theory with a $\theta$ term}

The Euclidean action of the two-dimensional pure U(1) lattice gauge theory with a $\theta$ term is defined by

$$
\begin{aligned}
S & =-\beta \sum_{x} \cos p_{x}-i \theta Q, \\
p_{x} & =\varphi_{x, 1}+\varphi_{x+\hat{1}, 2}-\varphi_{x+\hat{2}, 1}-\varphi_{x, 2}, \\
Q & =\frac{1}{2 \pi} \sum_{x} q_{x}, \quad q_{x}=p_{x} \bmod 2 \pi,
\end{aligned}
$$

where $\varphi_{x, \mu} \in[-\pi, \pi]$ is the phase of $\mathrm{U}(1)$ link variable at site $x$ in $\mu$ direction. The range of $q_{x}$ is $[-\pi, \pi]$ and it can be expressed as follows by introducing an integer $n_{x}$ :

$$
q_{x}=p_{x}+2 \pi n_{x}, \quad n_{x} \in\{-2,-1,0,1,2\} .
$$

For the periodic boundary condition, the topological charge $Q$ becomes an integer:

$$
Q=\sum_{x}\left(\frac{p_{x}}{2 \pi}+n_{x}\right)=\sum_{x} n_{x}
$$

The tensor may be given with continuous indices,

$$
\mathcal{T}\left(\varphi_{x, 1}, \varphi_{x+\hat{1}, 2}, \varphi_{x+\hat{2}, 1}, \varphi_{x, 2}\right)=\exp \left(\beta \cos p_{x}+i \frac{\theta}{2 \pi} q_{x}\right) .
$$

\footnotetext{
${ }^{1}$ See ref. [21] for recent studies of the Schwinger model with the $\theta$ term in the Hamiltonian formalism.
} 
The partition function is represented as

$$
Z=\left(\prod_{x, \mu} \int_{-\pi}^{\pi} \frac{d \varphi_{x, \mu}}{2 \pi}\right) \prod_{x} \mathcal{T}\left(\varphi_{x, 1}, \varphi_{x+\hat{1}, 2}, \varphi_{x+\hat{2}, 1}, \varphi_{x, 2}\right) .
$$

\subsection{Gauss-Legendre quadrature method}

In order to obtain a finite dimensional tensor network, we discretize all the integrals in eq. (2.7) using a numerical quadrature. In general, an integral of a function $f(\varphi)$ can be evaluated by

$$
\int d \varphi f(\varphi) \approx \sum_{\alpha=1}^{K} w_{\alpha} f\left(\varphi^{(\alpha)}\right)
$$

where $\varphi^{(\alpha)}$ and $w_{\alpha}$ are the $\alpha$-th node of the $K$-th polynomial and the associated weight, respectively. In this work, we use the Gauss-Legendre quadrature for discretization. The discretized local tensor can be expressed as

$$
T_{i j k l}=\frac{\sqrt{w_{i} w_{j} w_{k} w_{l}}}{(2 \pi)^{2}} \mathcal{T}\left(\varphi^{(i)}, \varphi^{(j)}, \varphi^{(k)}, \varphi^{(l)}\right)
$$

and we get a finite dimensional tensor network

$$
Z \approx \sum_{\{\alpha\}} \prod_{x} T_{\alpha_{x, 1} \alpha_{x+\hat{1}, 2} \alpha_{x+\hat{2}} \alpha_{x, 2}}
$$

where $\{\alpha\}$ represents a set of indices associated with the Gauss-Legendre quadrature. ${ }^{2}$

\subsection{Improved method}

We have developed further improvement for the above method. In the singular value decomposition (SVD) procedure to prepare the initial tensor before starting the iterative TRG steps $[12,16,19]$, we employ the following eigenvalue decomposition:

$$
M_{i j k l}=\frac{\sqrt{w_{i} w_{j} w_{k} w_{l}}}{(2 \pi)^{4}} \int_{-\pi}^{\pi} d \varphi_{1} d \varphi_{2} \mathcal{T}\left(\varphi^{(i)}, \varphi^{(j)}, \varphi_{1}, \varphi_{2}\right) \mathcal{T}^{*}\left(\varphi^{(k)}, \varphi^{(l)}, \varphi_{1}, \varphi_{2}\right)
$$

which is essentially equivalent to

$$
M_{i j k l}=\lim _{K^{\prime} \rightarrow \infty} \sum_{m, n=1}^{K^{\prime}} T_{i j m n} T_{k l m n}^{*} .
$$

This procedure is expected to reduce the discretization errors in $M_{i j k l}$.

To evaluate eq. (2.11), we use the character expansion [24, 25]:

$$
\mathcal{T}\left(\varphi_{1}, \varphi_{2}, \varphi_{3}, \varphi_{4}\right)=\sum_{m, n=-\infty}^{\infty} e^{i n\left(\varphi_{1}+\varphi_{2}-\varphi_{3}-\varphi_{4}\right)} I_{m}(\beta) J_{n-m}(\theta)
$$

\footnotetext{
${ }^{2}$ Application of the plain Gauss-Legendre quadrature method to this model was originally proposed by Yuya Shimizu.
} 
where $I_{m}(\beta)$ is the $m$-th order modified Bessel function of the first kind and

$$
J_{n}(\theta)=(-1)^{n} \frac{2}{\theta+2 \pi n} \sin \left(\frac{\theta}{2}\right) .
$$

Then, eq. (2.11) is rewritten as

$$
M_{i j k l}=\frac{\sqrt{w_{i} w_{j} w_{k} w_{l}}}{(2 \pi)^{4}} \sum_{n=-\infty}^{\infty} e^{i n\left(\varphi^{(i)}+\varphi^{(j)}-\varphi^{(k)}-\varphi^{(l)}\right)}\left(\sum_{m, m^{\prime}=-\infty}^{\infty} I_{m}(\beta) I_{m^{\prime}}(\beta) J_{n-m}(\theta) J_{n-m^{\prime}}(\theta)\right) .
$$

In the practical calculation, the sums of $n, m$ and $m^{\prime}$ can be truncated when the contributions of the terms are small enough. In this work we discard the contributions of $I_{m, m^{\prime}} / I_{0}<10^{-12}$ or $J_{n-m, n-m^{\prime}} / J_{0}<10^{-12}$.

\section{Numerical analysis}

\subsection{Setup}

The partition function of eq. (2.7) is evaluated with the TRG method at $\beta=0.0$ and 10.0 as a function of $\theta$ on a $V=L \times L$ lattice, where $L$ is enlarged up to 1024 . We choose $K=32$ for the polynomial order of the Gauss-Legendre quadrature in eq. (2.8). The SVD procedure in the TRG method is truncated with $D=32$. We have checked that these choices of $D$ and $K$ provide us sufficiently converged results for all the parameter sets employed in this work. Since the scaling factor of the TRG method is $\sqrt{2}$, allowed lattice sizes for the partition function are $L=\sqrt{2}, 2,2 \sqrt{2}, \cdots, 512 \sqrt{2}, 1024$. The periodic boundary condition is employed in both directions so that the topological charge $Q$ is quantized to be an integer.

\subsection{Free energy}

The analytic result for the partition function of eq. (2.7) is given by [22]:

$$
\begin{aligned}
& Z_{\text {analytic }}=\sum_{Q=-\infty}^{\infty}\left(z_{\mathrm{P}}(\theta+2 \pi Q, \beta)\right)^{V}, \\
& z_{\mathrm{P}}(\theta, \beta)=\int_{-\pi}^{\pi} \frac{d \varphi_{\mathrm{P}}}{2 \pi} \exp \left(\beta \cos \varphi_{\mathrm{P}}+i \frac{\theta}{2 \pi} \varphi_{\mathrm{P}}\right),
\end{aligned}
$$

where $z_{\mathrm{P}}(\theta, \beta)$ denotes the one-plaquette partition function with $\varphi_{\mathrm{P}} \in[-\pi, \pi]$. In figure 1 we plot the magnitude of the relative error for the free energy defined by

$$
\delta f=\frac{\left|\ln Z_{\text {analytic }}-\ln Z(K, D=32)\right|}{\left|\ln Z_{\text {analytic }}\right|}
$$

at $\theta=\pi$ on a $1024 \times 1024$ lattice. There are a couple of important points to be noted. Firstly, the deviation quickly diminishes as $K$ increases even at $\theta=\pi$, around which the Monte Carlo approaches do not work effectively due to large statistical errors [26]. Secondly, 


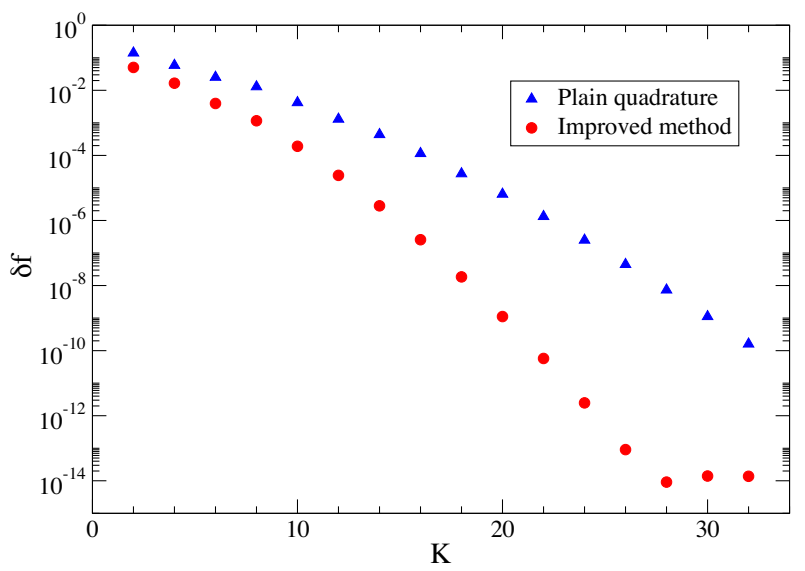

Figure 1. Relative error of free energy as a function of $K$ with $D=32$ on a $1024 \times 1024$ lattice. $K$ is the polynomial order of the Gauss-Legendre quadrature in eq. (2.8).

our method yields more precise results than the plain Gauss-Legendre quadrature method at any value of $K$. Thirdly, our choice of a parameter set of $(D, K)=(32,32)$ yields $\delta f<10^{-12}$, which means that the free energy is determined at sufficiently high precision. Hereafter we present the results obtained with $(D, K)=(32,32)$.

\subsection{Topological charge density}

The expectation value of the topological charge $\langle Q\rangle$ at $\beta=10.0$ is obtained by the numerical derivative of the free energy with respect to $\theta$ :

$$
\langle Q\rangle=-i \frac{\partial \ln Z}{\partial \theta}
$$

In figure 2 we show the volume dependence of $\langle Q\rangle / V$ around $\theta=\pi$, where the analytic calculation predicts the first order phase transition at any value of $\beta$ [22]. We observe that a finite discontinuity emerges with mutual crossings of curves between different volumes at $\theta=\pi$ as the lattice size $L$ is increased. This feature indicates there is a first order phase transition at $\theta=\pi$.

It may be interesting to calculate the topological charge density in the strong coupling limit $\beta=0.0$, whose analytical result was obtained by Seiberg in the infinite volume limit $[2]$ :

$$
\left.\frac{\langle Q\rangle}{V}\right|_{\beta=0}=-i\left(\frac{1}{2} \cot \left(\frac{\theta}{2}\right)-\frac{1}{\theta}\right)
$$

Figure 3 compares the numerical result at $\beta=0.0$ with the analytic expression of eq. (3.5). The discrepancy found around $\theta=\pi$ with small lattice size of $L=4$ essentially vanishes once we increase the lattice size up to $L=64$. 


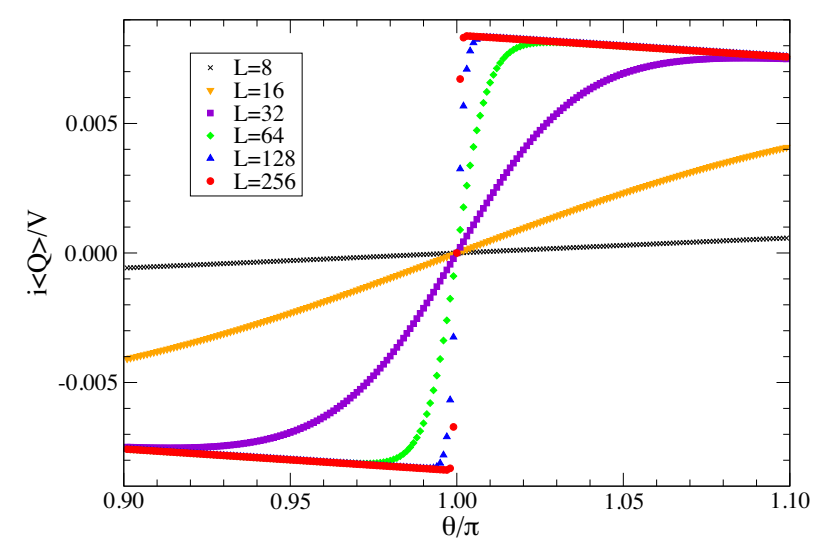

Figure 2. Topological charge density with $8 \leq L \leq 256$ as a function of $\theta$ at $\beta=10.0$.

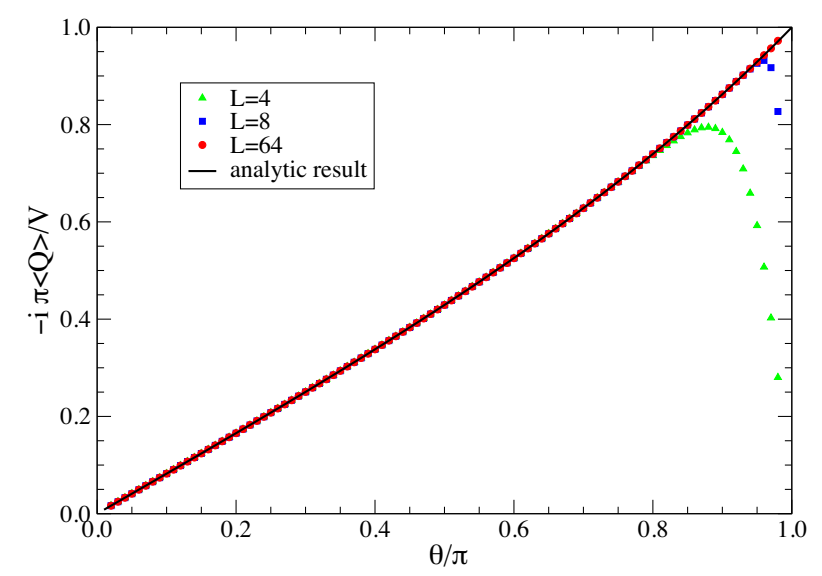

Figure 3. Topological charge density with $4 \leq L \leq 64$ as a function of $\theta$ at $\beta=0.0$. Solid curve denotes the analytic result of eq. (3.5) obtained in the infinite volume limit.

\subsection{Topological susceptibility}

We investigate the properties of the phase transition by applying the finite size scaling analysis to the topological susceptibility:

$$
\chi(L)=-\frac{1}{V} \frac{\partial^{2} \ln Z}{\partial \theta^{2}} .
$$

Figure 4 shows the topological susceptibility as a function of $\theta$ for various lattice sizes. The peak structure is observed and its height $\chi_{\max }(L)$ grows as $L$ increases. In order to determine the peak position $\theta_{c}(L)$ and the peak height $\chi_{\max }(L)$ at each $L$, we employ the quadratic approximation of the topological susceptibility around the peak position:

$$
\chi(L) \sim \chi_{\max }(L)+R\left(\theta-\theta_{c}(L)\right)^{2}
$$

with $\mathrm{R}$ a constant.

We expect that the peak height scales with $L$ as

$$
\chi_{\max }(L) \propto L^{\gamma / \nu},
$$




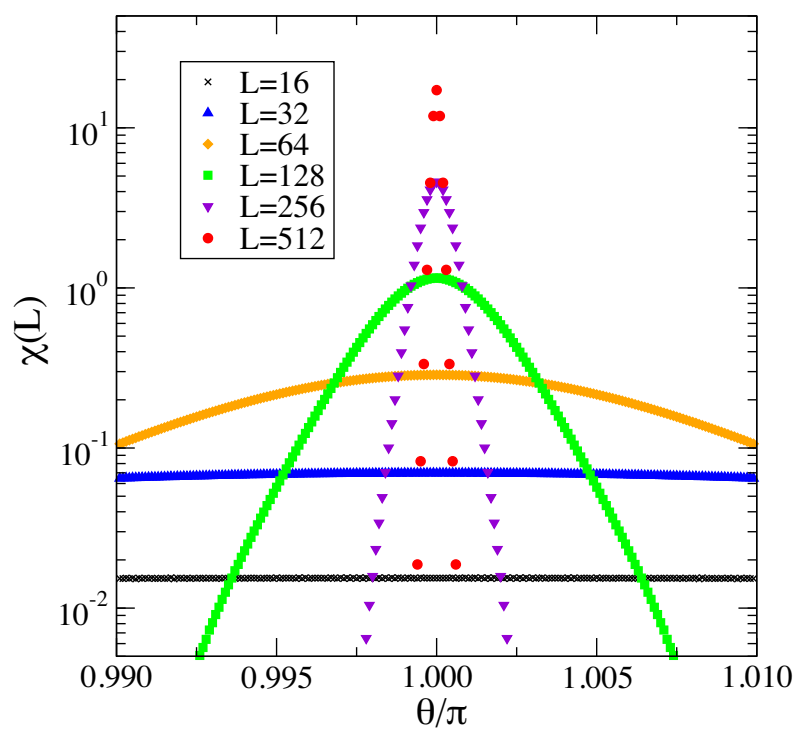

Figure 4. Topological susceptibility $\chi(L)$ as a function of $\theta$ with $16 \leq L \leq 512$.

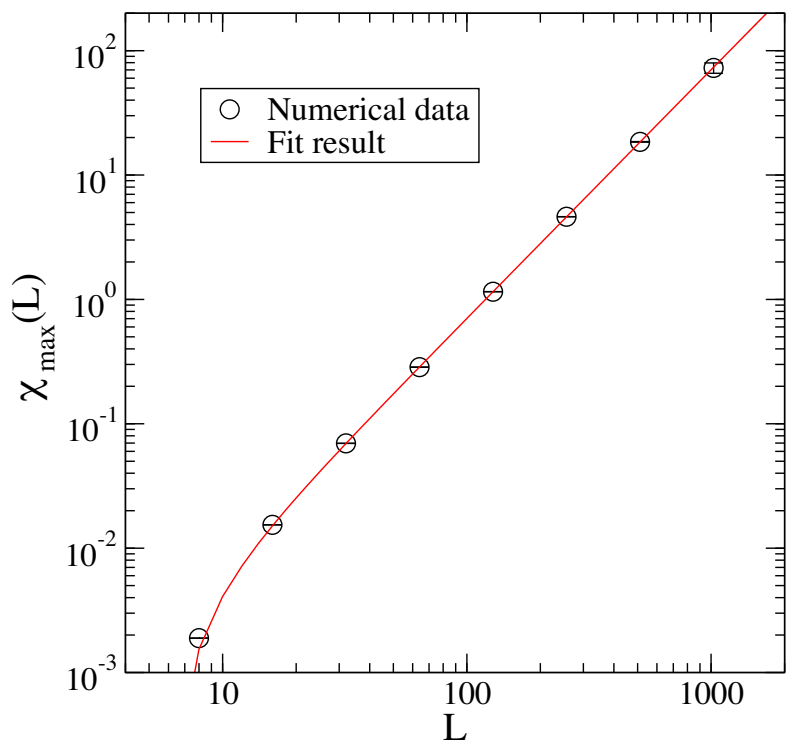

Figure 5. Peak height of topological susceptibility $\chi_{\max }(L)$ as a function of $L$. Solid curve denotes the fit result.

where $\gamma$ and $\nu$ are the critical exponents. The $L$ dependence of the peak height $\chi_{\max }(L)$ is plotted in figure 5 . The solid curve represents the fit result obtained with the fit function of $\chi_{\max }(L)=A+B L^{\gamma / \nu}$ choosing the fit range of $128 \leq L \leq 1024$. The results for the fit parameters are given by $A=-3(2) \times 10^{-3}, B=7.12(8) \times 10^{-5}$ and $\gamma / \nu=1.998(2)$. The value of the exponent $\gamma / \nu=1.998(2)$ is consistent with two, which is the expected critical exponent in the first-order phase transition in the two-dimensional system. 


\section{Summary and outlook}

We have applied the TRG method to study the $2 \mathrm{D}$ pure $\mathrm{U}(1)$ gauge theory with a $\theta$ term. The continuous degrees of freedom are discretized with the Gauss quadrature method. We have confirmed that this model has a first-order phase transition at $\theta=\pi$ as predicted from the analytical calculation. The successful analysis of the model demonstrates an effectiveness of the Gauss quadrature approach to the gauge theories. It should be interesting to apply the TRG-based methods with the Gauss quadrature to higher dimensional gauge theories with $\theta$ term which have been hardly investigated by the Monte Carlo approach because of the sign problem. Another interesting research direction is to include fermionic degrees of freedom following the Grassmann TRG method developed in ref. [7]. This is a necessary ingredient toward investigation of the phase structure of QCD at finite density.

\section{Acknowledgments}

One of the authors (YK) thanks Yuya Shimizu for providing the results obtained by the plain Gauss-Legendre quadrature method. Numerical calculation for the present work was carried out with the Cygnus computer under the Interdisciplinary Computational Science Program of Center for Computational Sciences, University of Tsukuba. This work is supported by the Ministry of Education, Culture, Sports, Science and Technology (MEXT) as "Exploratory Challenge on Post-K computer (Frontiers of Basic Science: Challenging the Limits)".

Open Access. This article is distributed under the terms of the Creative Commons Attribution License (CC-BY 4.0), which permits any use, distribution and reproduction in any medium, provided the original author(s) and source are credited.

\section{References}

[1] D.J. Gross and E. Witten, Possible Third Order Phase Transition in the Large N Lattice Gauge Theory, Phys. Rev. D 21 (1980) 446 [INSPIRE].

[2] N. Seiberg, Topology in Strong Coupling, Phys. Rev. Lett. 53 (1984) 637 [InSPIRE].

[3] E. Witten, Theta dependence in the large $N$ limit of four-dimensional gauge theories, Phys. Rev. Lett. 81 (1998) 2862 [hep-th/9807109] [INSPIRE].

[4] D. Gaiotto, A. Kapustin, Z. Komargodski and N. Seiberg, Theta, Time Reversal and Temperature, JHEP 05 (2017) 091 [arXiv: 1703.00501] [INSPIRE].

[5] M. Levin and C.P. Nave, Tensor renormalization group approach to $2 D$ classical lattice models, Phys. Rev. Lett. 99 (2007) 120601 [cond-mat/0611687] [INSPIRE].

[6] Y. Shimizu, Tensor renormalization group approach to a lattice boson model, Mod. Phys. Lett. A 27 (2012) 1250035 [INSPIRE].

[7] Y. Shimizu and Y. Kuramashi, Grassmann tensor renormalization group approach to one-flavor lattice Schwinger model, Phys. Rev. D 90 (2014) 014508 [arXiv:1403.0642] [INSPIRE]. 
[8] Y. Shimizu and Y. Kuramashi, Critical behavior of the lattice Schwinger model with a topological term at $\theta=\pi$ using the Grassmann tensor renormalization group, Phys. Rev. D 90 (2014) 074503 [arXiv: 1408.0897] [INSPIRE].

[9] Y. Shimizu and Y. Kuramashi, Berezinskii-Kosterlitz-Thouless transition in lattice Schwinger model with one flavor of Wilson fermion, Phys. Rev. D 97 (2018) 034502 [arXiv: 1712.07808] [inSPIRE].

[10] S. Takeda and Y. Yoshimura, Grassmann tensor renormalization group for the one-flavor lattice Gross-Neveu model with finite chemical potential, PTEP 2015 (2015) 043B01 [arXiv: 1412.7855] [INSPIRE].

[11] H. Kawauchi and S. Takeda, Tensor renormalization group analysis of $C P(N-1)$ model, Phys. Rev. D 93 (2016) 114503 [arXiv: 1603.09455] [INSPIRE].

[12] D. Kadoh, Y. Kuramashi, Y. Nakamura, R. Sakai, S. Takeda and Y. Yoshimura, Tensor network formulation for two-dimensional lattice $\mathcal{N}=1$ Wess-Zumino model, JHEP 03 (2018) 141 [arXiv : 1801.04183] [INSPIRE].

[13] R. Sakai, S. Takeda and Y. Yoshimura, Higher order tensor renormalization group for relativistic fermion systems, PTEP 2017 (2017) 063B07 [arXiv:1705.07764] [INSPIRE].

[14] Y. Yoshimura, Y. Kuramashi, Y. Nakamura, S. Takeda and R. Sakai, Calculation of fermionic Green functions with Grassmann higher-order tensor renormalization group, Phys. Rev. D 97 (2018) 054511 [arXiv:1711.08121] [INSPIRE].

[15] J. Unmuth-Yockey, J. Zhang, A. Bazavov, Y. Meurice and S.-W. Tsai, Universal features of the Abelian Polyakov loop in 1+1 dimensions, Phys. Rev. D 98 (2018) 094511 [arXiv: 1807.09186] [INSPIRE].

[16] D. Kadoh, Y. Kuramashi, Y. Nakamura, R. Sakai, S. Takeda and Y. Yoshimura, Tensor network analysis of critical coupling in two dimensional $\phi^{4}$ theory, JHEP 05 (2019) 184 [arXiv: 1811.12376] [INSPIRE].

[17] Y. Kuramashi and Y. Yoshimura, Three-dimensional finite temperature $Z_{2}$ gauge theory with tensor network scheme, JHEP 08 (2019) 023 [arXiv: 1808.08025] [INSPIRE].

[18] N. Butt, S. Catterall, Y. Meurice and J. Unmuth-Yockey, Tensor network formulation of the massless Schwinger model, arXiv:1911.01285 [INSPIRE].

[19] D. Kadoh, Y. Kuramashi, Y. Nakamura, R. Sakai, S. Takeda and Y. Yoshimura, Investigation of complex $\phi^{4}$ theory at finite density in two dimensions using TRG, JHEP 02 (2020) 161 [arXiv: 1912.13092] [INSPIRE].

[20] S.R. Coleman, More About the Massive Schwinger Model, Annals Phys. 101 (1976) 239 [INSPIRE].

[21] L. Funcke, K. Jansen and S. Kühn, Topological vacuum structure of the Schwinger model with matrix product states, Phys. Rev. D 101 (2020) 054507 [arXiv:1908.00551] [INSPIRE].

[22] U.J. Wiese, Numerical Simulation of Lattice $\theta$ Vacua: The 2-d U(1) Gauge Theory as a Test Case, Nucl. Phys. B 318 (1989) 153 [InSPIRE].

[23] Y. Liu et al., Exact Blocking Formulas for Spin and Gauge Models, Phys. Rev. D 88 (2013) 056005 [arXiv: 1307.6543] [INSPIRE].

[24] A.S. Hassan, M. Imachi and H. Yoneyama, Real space renormalization group analysis of U(1) gauge theory with Theta term in two-dimensions, Prog. Theor. Phys. 93 (1995) 161 [hep-lat/9410003] [INSPIRE]. 
[25] A.S. Hassan, M. Imachi, N. Tsuzuki and H. Yoneyama, Character expansion, zeros of partition function and theta term in U(1) gauge theory, Prog. Theor. Phys. 94 (1995) 861 [hep-lat/9508011] [INSPIRE].

[26] J.C. Plefka and S. Samuel, Monte Carlo studies of two-dimensional systems with a theta term, Phys. Rev. D 56 (1997) 44 [hep-lat/9704016] [INSPIRE]. 